\title{
Improvement of quality of details at round grinding in the conditions of a floating workshop
}

\author{
Sergey Bratan ${ }^{1}$, Ekaterina Vladetskaya ${ }^{1}$, and Aleksander Kharchenko ${ }^{1, *}$ \\ ${ }^{1}$ Sevastopol State University, Sevastopol, Russian Federation
}

\begin{abstract}
The results of laboratory studies of the grinding processing process are given taking into account the evaluation of the quality of the surfaces of the shafts with disturbing vibrational influences on the equipment of external forces that are the consequence of sea waves, as well as the neighboring operating equipment in a floating workshop. To determine the influence of external influences on the quality of processing during grinding, an experimental stand was used, including a cylindrical grinding machine with a complex of test equipment, as well as a compressor placed at a different distance from the machine, creating external vibration effects simulating external disturbances through the surface Floor and foundation on the machine bed, corresponding to the effects of the external environment and the operating neighboring equipment. The dependences of the frequency of mechanical oscillations (in the measuring zones-the basebed and the machine table guides in the transverse and vertical directions) are obtained, the amplitude of the vibro-displacement in these zones from the conditions of the circular grinding machine and sea-wave levels. The changes in the error in the shape of the machined parts, the roughness of the machined surface, the waviness of the roll neck surfaces according to the basic and achieved variants are determined taking into account the use of new designs of effective vibration isolating supports and devices.
\end{abstract}

\section{Relevance of the problem}

Existence of such mobile mini-plants as floating workshops and road floating workshops with the production sites placed on decks including machining, having a wide range of the machine equipment, including grinders is characteristic of the industry of the seaside cities. Such workshops are capable to perform complex works on production and modernization of hitech products, knots and units in areas, remote from the main stationing sites. Feature of operation of the specified equipment is existence of the vibrations caused by impact on him of various sources reducing accuracy and raising a roughness of the processed surfaces during technological process [1].

Improvement of quality of processing of details in the conditions of floating workshops is directly connected with need of reduction of the compelled fluctuations of the machine transmitted through the surface of the deck from external sources including from influences of the external environment - disorders of a surface of the water. As a rule, on decks of floating workshops fixing of machines is provided with the base which doesn't provide sufficient vibration insulation owing to considerable external oscillatory influences, poorly studied so far. Their influence on quality of processing on the finishing operations complicated by oscillatory processes of different level wasn't investigated. In works $[2,3]$ modeling of interaction of the tool and the processed preparation in the conditions of floating workshops is executed. The dynamic system with difficult stationary and non-stationary vibration influences, and also shock influences from the external equipment and long sea nervousness through floating foundation and the surface of the deck is considered. On the basis of the systems concept the structure of operation is offered, basic provisions and methods of the analysis of process are formulated; input, output variables and parameters of a status of each of subsystems are defined.

To research of processes of grinding of necks of shaft for the purpose of determination of parameters of quality of the processed surfaces received at various levels of external influences under production conditions (fig. 1) and at the experimental stand (fig. 2) in laboratory works are devoted $[4,5]$.

On the basis of the morphological analysis and synthesis of structures of anti-vibration devices the morphological matrix is constructed, signs and characteristics of subsystems and elements are considered, communications between them are revealed. Options of structures of the anti-vibration device taking into account the essential signs and technical requirements defining properties of system are synthesized. Structural and layout optimization of antivibration devices is executed. Parametrical synthesis, theoretical and pilot studies of real designs of antivibration devices have led to creation of the new antivibration device (fig. 3,a) the machine of a floating

\footnotetext{
Corresponding author: khao@list.ru
} 
workshop on the basis of a support (fig. 3, b) with rubber-metal and hydraulic dampers.

The purpose of this article is development of practical recommendations about improvement of quality of grinding of details in the conditions of floating workshops on the basis of results of tests of new antivibration devices in the conditions similar to conditions of a floating workshop.

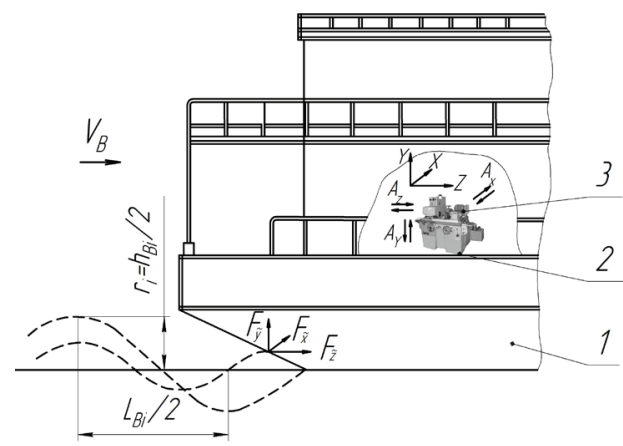

Fig. 1. Scheme of an arrangement the circular grinding machine on the site of a floating workshop: 1 - the floating basis, 2 - the surface of the deck, 3 - the machine $3 \mathrm{~B} 12$

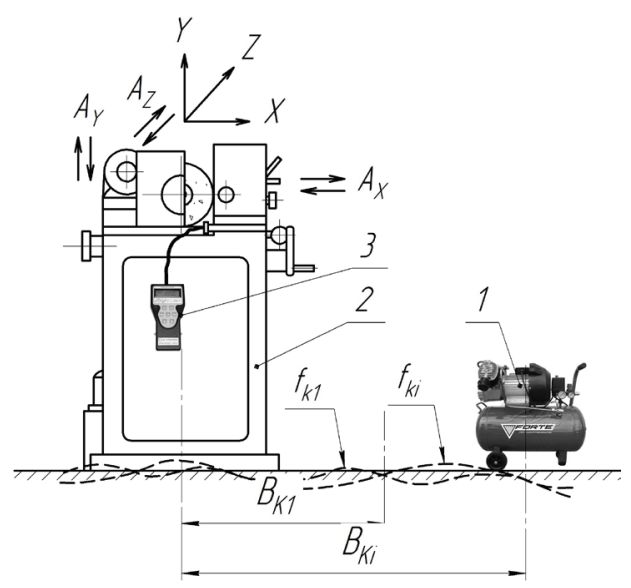

Fig. 2. Scheme of experimental the stand for imitation of the external vibration influences when grinding arising on a floating workshop: 1 - FINI Tiger 245 compressor; 2 - BUA 16 machine; 3 - «Vibrotest-MG4.01» vibrometer

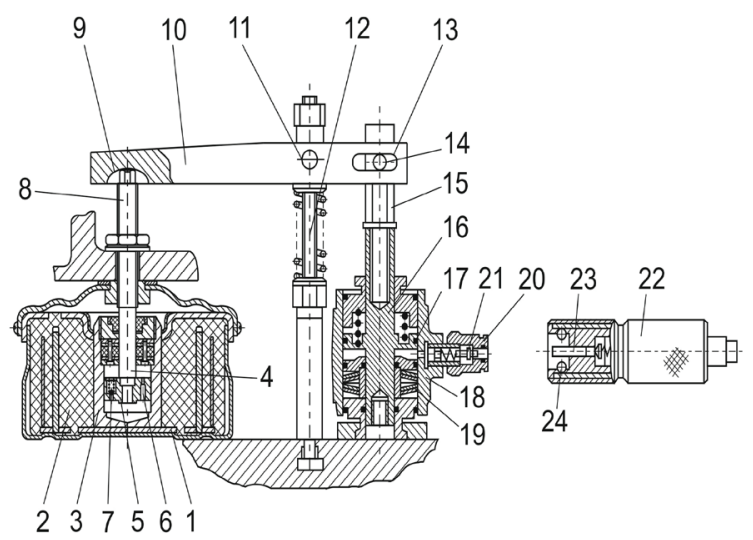

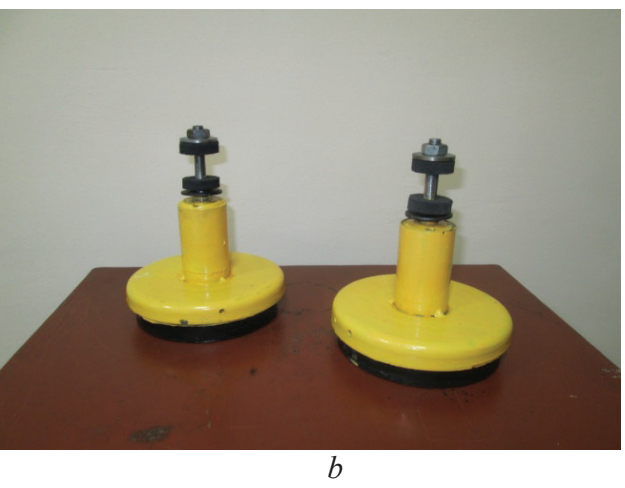

Fig. 3. A general view of a new design of the anti-vibration device $(a)$ on the basis of a support $(b)$ with rubber-metal and hydraulic dampers

\section{Technique of carrying out researches}

For definition of influence of external impacts on quality of processing when grinding used the laboratory equipment (fig. 2) as a part of the experimental stand which is turning on the circular grinding BUA 16 machine equipped with a control instrumentation complex. The compressor 1 (FINI Tiger 245) placed on different removal $(B k 1 \ldots B k i)$ from the machine 2, creating during the work on the different modes the external vibration impacts on technological system of the machine $(f k 1$... $f k i)$ imitating external indignations through a surface of a floor and the base on a machine bed. They correspond to impacts on the machine in a buoyant workshop of an external environment (sea disturbance) and the operating adjacent equipment, causing vibrations and vibration displacements with amplitudes of $A_{X}, A_{Y}, A_{Z}$ respectively in the directions of its axes of coordinates. For determination of parameters of vibrations and amplitude of vibration displacements used the vibrometer 3 «Vibrotest-MG4.01». The carriedout stage of imitating modeling has allowed to define the main conditions under which carrying out grinding processing on the laboratory equipment with receiving the indicators of quality corresponding to these or those conditions of external influences (nervousness level, operation of the external equipment) on round grinding in a floating workshop [6] is possible. By the developed technique and under the set conditions carried out on the circular grinding machine processing of surfaces of necks of shaft $\left(d=26,00_{-0,025}^{+0,050}, \mathrm{~mm}\right)$ for kits of parts, imitating various levels of disorders and at the same time existence or lack of the working external equipment for each of parties.

For carrying out tests of new designs at the experimental stand (fig. 2) the machine was installed on anti-vibration support of a new design (fig.4), other processing and control and measuring equipment imitated in vitro different influences for sea nervousness and from work of the next objects. 


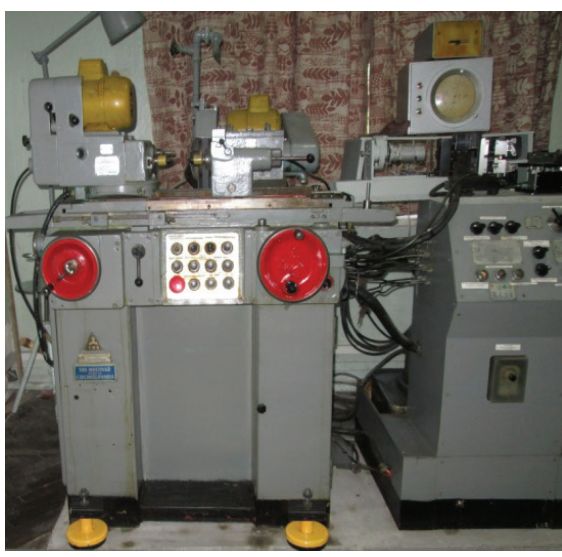

Fig.4. The circular grinding BUA 16 machine on anti-vibration support of a new design (UA 36389 patent)

\section{Main content of work}

Results of comparative tests on indicators of vibration impacts on the circular grinding machine at installation on the base (basic option) or on anti-vibration support (the reached option) are received in vitro, corresponding to conditions of a floating workshop. Dependences of frequencies of mechanical oscillations ( $\left.f k x, f k y, f^{\prime} o x, f o y\right)$ from statuses of the circular grinding machine and level of disturbance $h_{3 \%}$ are illustrated with diagrams on figs. 5. Lowering of frequency of mechanical oscillations (fig.5, a) foy in the lower part of a bed in the vertical direction (along a $\mathrm{Y}$ axis) on average from 1,4 times (in case of the rough sea and the inoperable machine) to 2,5 times in case of the rough sea and the operating machine. Vibromovements of Sox in the lower part of a bed in the cross direction (along axis X) decrease on average from 2,14 times (at a rough sea and the working machine) to 3,74 times at a rough sea and the idle machine. Vibromovements of Soy in the lower part of a bed in the longitudinal direction (along axis $\mathrm{Y}$ ) decrease on average from 1,1 times (at weak nervousness and the idle machine) to 1,8 times at a rough sea and the working machine.

The analysis of indices in case of installation of the machine on anti-vibration support of new construction shows lowering of frequency of mechanical oscillations of $f^{\prime}$ 'ox in a zone of guides of the machine in the cross direction (along a $\mathrm{X}$ axis) on average from 1,8 times (in case of the considerable and strong disturbances of both the operating machine, and at the same time adjacent equipment and cooling) to 2,1 times in case of feeble disturbance (all three statuses of the machine) and the operating machine in case of the rough sea. Lowering of frequency of mechanical oscillations of $f^{\prime} \mathrm{oy}$ in a zone of guides of the machine in the vertical direction (along a Y axis) from 1,5 times (in case of the rough sea and the inoperable machine) to 1,8 times in case of feeble disturbance and the inoperable machine. Vibromovements (fig. $5, b$ ) $S^{\prime} x$ in a zone of guides of the machine in the cross direction (along axis X) decrease on average from 2,3 times (at strong nervousness and the working machine along with the next equipment and ventilation) to 3,8 times at weak nervousness and the idle machine. Vibromovements of $S^{\prime} y$ in a zone of guides of the machine in the longitudinal direction (along axis $\mathrm{Y}$ ) decrease on average from 1,7 times (at strong nervousness and the working machine along with the next equipment and ventilation) to 2,9 times at a rough sea and the working machine.

When performing circular grinding processing of surfaces of necks of shaft results of quality check of the surfaces processed at the same time are received (table $1)$.
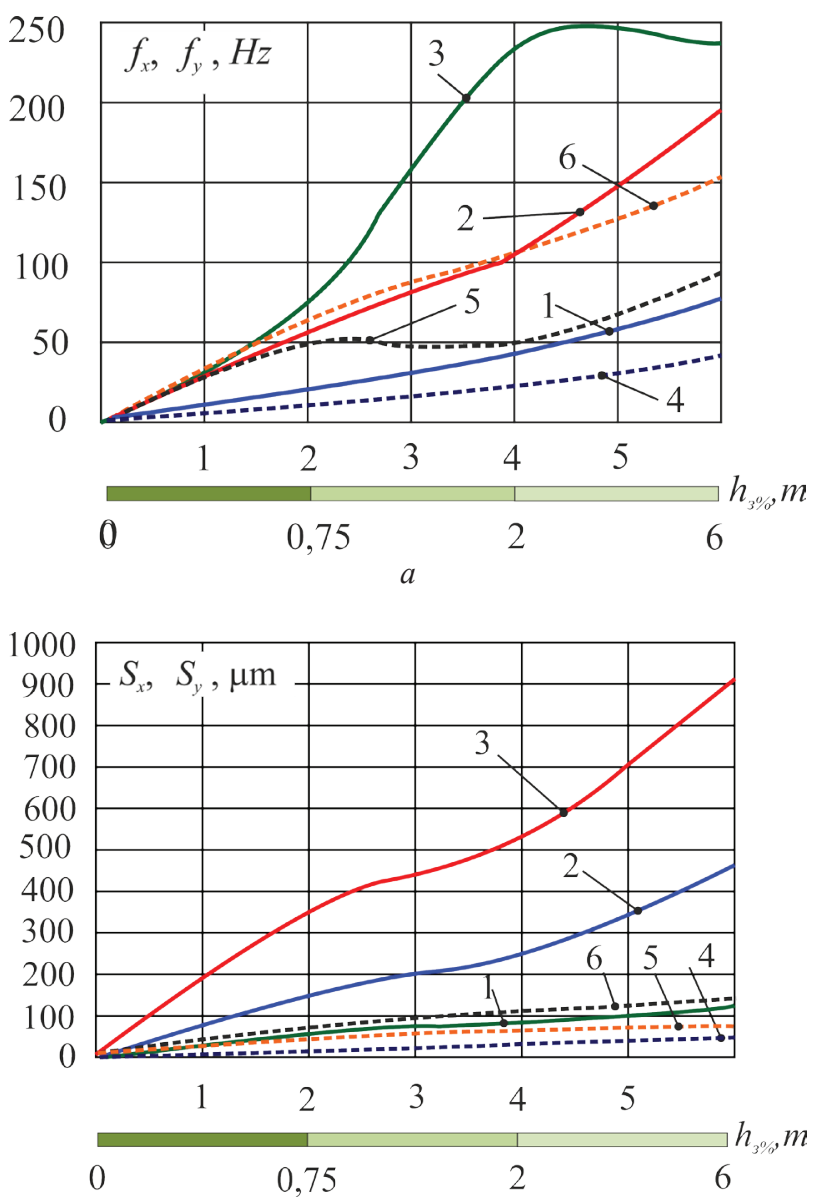

Fig. 5. Diagrams of dependences of oscillation frequency ( $f x$, $f y$ ) in a measurement zone a bed base $(a)$ and amplitudes of vibration displacement $\left(S^{\prime} x, S^{\prime} y\right)$ on guides of the desktop of the machine $(b)$ in case of different statuses of the circular grinding machine $(1,4-$ it is disconnected; 2, 5-works; 3, 6 - works along with the face grinding machine and cooling) from disturbance level 
Table 1. Comparison of results of quality of surfaces of necks of the shaft processed by the basic and reached options

\begin{tabular}{|c|c|c|c|c|c|}
\hline \multicolumn{3}{|c|}{$\begin{array}{l}\text { Beating of basic necks - } \\
\Delta, \mathrm{mkm} \text { (basic / reached) }\end{array}$} & \multicolumn{3}{|c|}{$\begin{array}{l}\text { Roughness of surface }- \\
R_{a}, m k m \text { (basic / reached) }\end{array}$} \\
\hline $\max$ & $\min$ & Dispersion $\sigma^{2}, \mathrm{mkm}^{2}$ & $\max$ & $\min$ & Dispersion $\sigma^{2}, \mathrm{mkm}^{2}$ \\
\hline \multicolumn{6}{|c|}{ Weak waves } \\
\hline $0,025 / 0,016$ & $0,010 / 0,011$ & $6,2 \times 10^{-6} / 0,7 \times 10^{-6}$ & $0,63 / 0,50$ & $0,41 / 0,32$ & $1,3 \times 10^{-3} / 0,9 \times 10^{-3}$ \\
\hline $0,048 / 0,029$ & $0,009 / 0,008$ & $4,2 \times 10^{-5} / 1,2 \times 10^{-5}$ & $0,80 / 0,63$ & $0,51 / 0,42$ & $2,3 \times 10^{-3} / 1,2 \times 10^{-3}$ \\
\hline \multicolumn{6}{|c|}{ Significant waves } \\
\hline $0,094 / 0,052$ & $0,018 / 0,015$ & $15,9 \times 10^{-5} / 3,8 \times 10^{-5}$ & $1,00 / 0,82$ & $0,46 / 0,54$ & $8,1 \times 10^{-3} / 2,2 \times 10^{-3}$ \\
\hline $0,123 / 0,079$ & $0,008 / 0,017$ & $3,7 \times 10^{-6} / 1,1 \times 10^{-6}$ & $1,25 / 1,02$ & $0,50 / 0,48$ & $15,6 \times 10^{-3} / 8,1 \times 10^{-3}$ \\
\hline \multicolumn{6}{|c|}{ High waves } \\
\hline $0,124 / 0,069$ & $0,020 / 0,014$ & $2,9 \times 10^{-6} / 8,4 \times 10^{-5}$ & $1,6 / 1,25$ & $0,50 / 0,52$ & $3,3 \times 10^{-2} / 1,5 \times 10^{-2}$ \\
\hline $0,162 / 0,097$ & $0,023 / 0,018$ & $5,4 \times 10^{-4} / 1,7 \times 10^{-4}$ & $2,0 / 1,58$ & $0,63 / 0,62$ & $5,2 \times 10^{-2} / 2,6 \times 10^{-2}$ \\
\hline \multicolumn{6}{|c|}{ Waveform indices } \\
\hline \multicolumn{6}{|c|}{ Weak waves } \\
\hline \multicolumn{2}{|c|}{$W_{\max }, \mathrm{mkm}$} & $W_{z}, m k m$ & \multicolumn{2}{|l|}{$S_{w}, M \kappa M$} & $L_{w}, M \kappa M$ \\
\hline \multicolumn{2}{|c|}{$5,6 / 5,1$} & $4,8 / 4,2$ & \multicolumn{2}{|l|}{$192 / 169$} & $960 / 845$ \\
\hline \multicolumn{2}{|c|}{$7,2 / 5,8$} & $5,3 / 3,9$ & $265 / 211$ & & $1457,5 / 1170$ \\
\hline \multicolumn{6}{|c|}{ Significant waves } \\
\hline \multicolumn{2}{|c|}{$6,9 / 5,7$} & $5,4 / 4,2$ & $297 / 264$ & & $1782 / 1420$ \\
\hline \multicolumn{2}{|c|}{$10,2 / 6,1$} & $5,8 / 5,0$ & $916 / 870$ & & $4580 / 4350$ \\
\hline \multicolumn{6}{|c|}{ High waves } \\
\hline \multicolumn{2}{|c|}{$15,4 / 7,8$} & $9,9 / 6,1$ & $574 / 526$ & & $3920 / 2530$ \\
\hline \multicolumn{2}{|c|}{$23,8 / 10,2$} & $10,6 / 7,4$ & $1328 / 1170$ & & $6640 / 5850$ \\
\hline
\end{tabular}

The graphs $\Delta=f\left(h_{3 \%}\right)$ in fig. 6, a show that the difference in the deviation value of the shape of the roll neck surfaces $(\Delta \max -\Delta \min )$ treated in the base version is greater than in the achieved variant with vibration isolating supports, respectively, by an average of 2.3 times when the circular grinding machine is operated and the external equipment is idle (from 1.9 for strong waves, to 3 for weak waves) and for 1.82 times for a surface grinding machine and ventilation (from 1.76 for strong waves to 1.86 for weak ones). Also, the graphs (fig. 6, b) $R a=f\left(h_{3 \%}\right)$ of the roughness deviations for Ra max confirm the quality improvement with the use of new vibration-proof supports 1.25 times on average (both with external equipment and with a surfacegrinding Machine and ventilation, and for all levels of excitement, the improvement in quality by roughness is approximately the same - from 1.22 to 1.28 times). Changes in the indices for Ra min are not significant at that, although also the achieved variant gives a decrease in roughness averagely from 1.03 times (with the operation of the circular grinding machine and nonworking external equipment) to 1.08 times (when working simultaneously with the surface grinder and ventilation).

Dependence graphs $W=f\left(h_{3 \%}\right)$ of the waviness indices (fig.7,a) for $W \max$ and $W z$ of the roll neck surfaces from external influences and conditions of the circular grinding machine, as well as an example of the obtained profilogram in the basic version $(b)$ and using new vibration-isolating supports (c) show that the decrease in the waviness of Wmax averages 1.39 times (when a cylindrical grinding machine is used and external equipment is not working) to 1.75 times (with a working circular grinder simultaneously with a surface grinding machine and ventilation). Similarly, in the parameter $W z$, the decrease in the undulation with the use of new vibration-proof supports averages 1.35 times, respectively, for processing in the absence of external equipment and 1.32 times with a working circular grinder simultaneously with a surface grinding machine and ventilation.

Comparison of the obtained profilogram with the basic version of the treatment with the cylindrical grinding machine installed on the foundation (fig.7,b) with the achieved variant with the use of new vibration isolating supports (c) clearly illustrates the advantages of the measures developed and methods for synthesizing rational variants of vibration isolation devices.

Thus, the vibration-isolating supports (fig. 3, b) of patent UA 36389 confirmed the achievement of the stated goal in the process of testing - improving the quality of grinding when working in a floating workshop. However, in the test specimens of the supports for use in the floating workshop, some fastening elements are necessary, since the technological equipment located on the decks of the floating workshops is exposed to the danger of displacement due to the detachment of the support base from the surface of the deck. To do this, they must be securely fixed using a lever system on the rack and equipped with a hydraulic cylinder with a spring battery. Such vibration-proofing devices (patent UA 51621) adapted to the conditions of floating workshops (patent UA 51621), which are created on the basis of laboratory-tested supports, 
provide an improvement in the quality of grinding processing of parts by reducing the errors in their shape, reducing roughness and ripeness by reducing the levels of external and Internal vibrations, which are suppressed by vibration isolation supports and spring accumulators in hydraulic cylinders. In addition, it is possible to further modernize these devices in order to create automatic vibration protection and vibration isolation systems for grinding machines and other precision machines capable of modifying the damping properties of vibro-supports as a result of signals from vibration sensors during machining of parts in floating workshops.
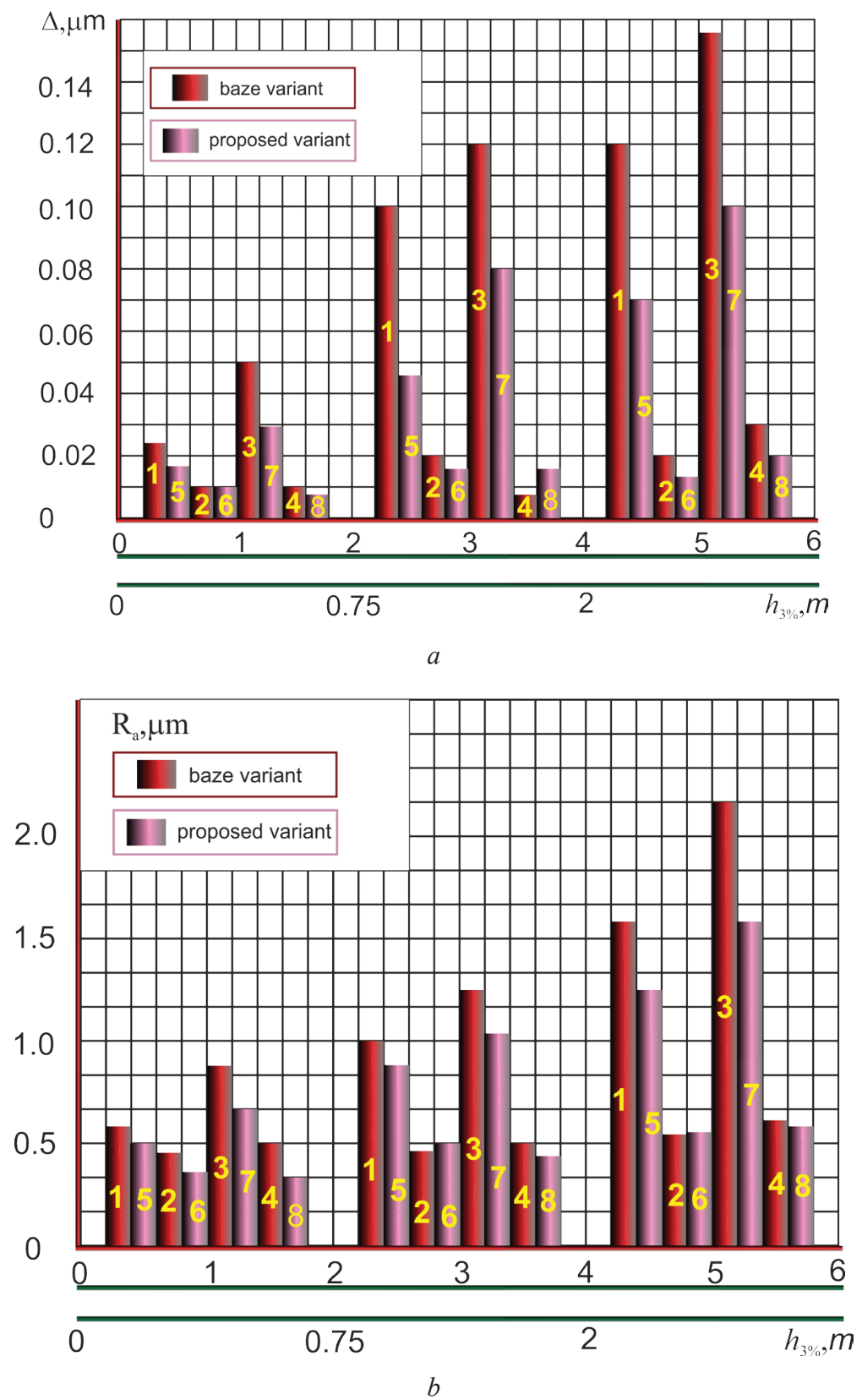

Fig. 6. Dependency curves of the deviations of the form $\Delta=f\left(h_{3} \%\right)$ for $\Delta \max (1,3$ - base, 5.7 - achieved variant) and $\Delta \min (2$, 4base, 6, 8 - achieved variant) $(a)$, and also the roughness $R a=f\left(h_{3 \%}\right)$ for $R a \max (1,3$ - basic, 5, 7 - the achieved variant) and $R a$ $\min (2,4$ - base, 6,8 - the achieved variant) $(b)$ shafts from external influences and conditions of the circular grinding machine $(1,2$, 5,6 - works, $3,4,7,8$ - works simultaneously with a surface grinding machine and ventilation) 


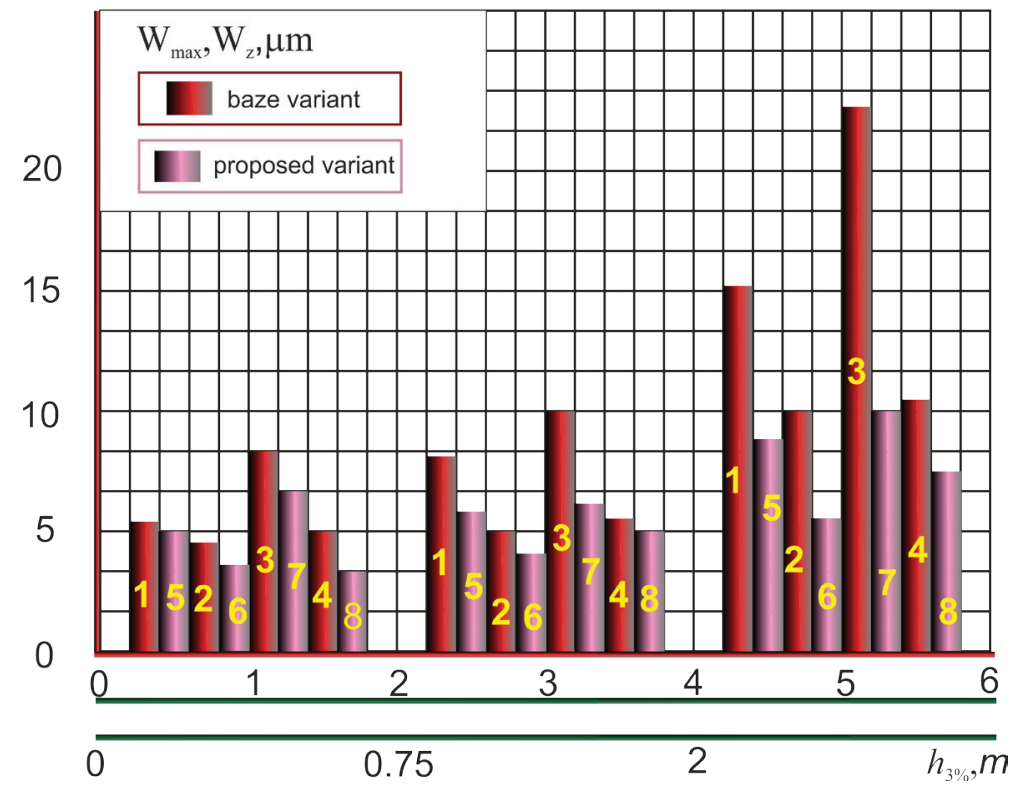

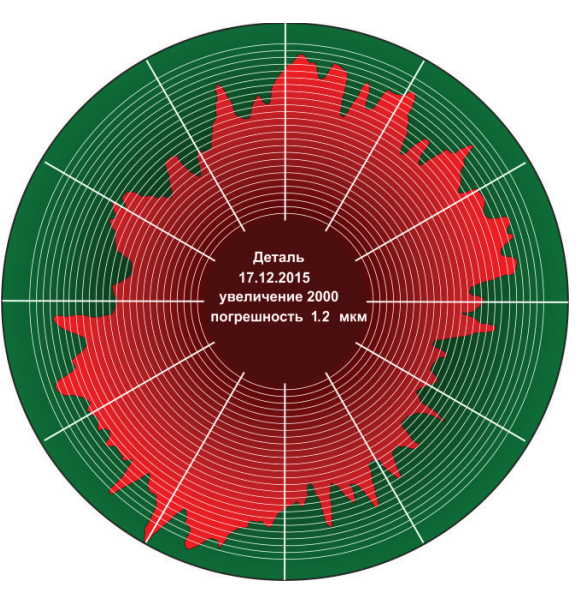

$b$ $a$

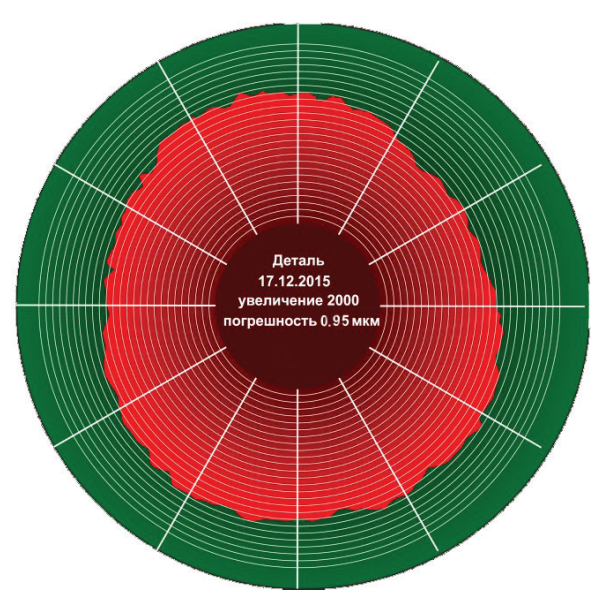

$c$

Fig. 7. Dependence graphs of the waviness indices $W=f\left(h_{3} \%\right)$ for $\operatorname{Wmax}(1,3$ - base, 5.7 - achieved variant) and $W z(2,4$ - base, 6 , 8 - achieved variant) $(a)$ shafts from external influences and conditions of the cylindrical grinding machine $(1,2,5,6$ - works; 3, 4, 7, 8 - works simultaneously with the surface grinding machine and ventilation), as well as an example of the roundogram obtained in the basic version $(b)$ and using new vibration-proof supports $(c)$

\section{Conclusions}

This approach allowed us to experimentally investigate the influence on the quality of parts during grinding processing of sea waves affecting the floating base of the workshop, revealed the expediency of using a new vibration protection system of the machine for solving the problems of ensuring dynamic stabilization of the grinding process $[1,2]$ and creating structures for effective vibration isolating supports and devices. The proposed constructions of vibration isolating supports and devices simplify the possibility of mounting and dismantling of technological equipment, contribute to improving the quality of machining of parts by reducing the errors of their shape by reducing external vibrational effects. The obtained results are the basis for further experimental studies of the parameters and characteristics of the technological system of a cylindrical grinding machine in the conditions of a floating workshop [6]. In addition to the abovementioned measures in the conditions of floating workshops, the use of high-precision CNC machines equipped with adaptive control systems based on the development of new models of optimal systems with a stochastic process controller is possible.

\section{References}

1. E.A. Vladetskaya, S.M. Bratan, A.O. Kharchenko, D.O. Vladetsky, Maintenance of quality of grinding processing by reduction of external disturbances in conditions of floating workshop, Fundamental and Applied Problems of Engineering and Technology, № 6(314), P.88-103 (2015)

2. E.A.Vladetskaya, Development of a shaping filter that simulates the dynamics of sea waves in a floating repair shop, Bulletin of SevNTU, №.150, pp. 36-40 (2014) 
3. A. Kharchenko, S. Bratan, E. Vladetskaya, Increasing the accuracy of the round grinding process by efficient vibration isolation of the machin, International Scientific Conference on engineering design and research of automotive vehicles and machines «SAKON'08», P. 47-58 (2008)

4. A. Kharchenko, E. Vladetskaya, V. Dolgin, S. Bratan, Reaction of the dynamic system to an arbitrary signal by the example of a machine in the conditions of a floating workshop, Monografie (Lublin, Polska: Politechnika Lubelska, 2015)

5. S.M. Bratan, E.A. Vladetskaya, Investigation of the reliability of a vibration-isolating device of a grinding machine, Science intensive technologies in mechanical engineering, № 9 (63), p. 10-15 (2016)

6. E.A. Vladetskaya, Modeling vibration exposure from external sources during grinding of details in laboratory conditions, Jornal of modern technologies: Collection of scientific papers Sevastopol: SevGU, № 4, P.25-34 (2016) 